\title{
A New Biomedical Image Denoising method using an adaptive multi- resolution technique
}

\author{
LALIT MOHAN SATAPATHY ${ }^{1}$, PRANATI DAS ${ }^{2}$, \\ ${ }^{1}$ Department of EEE, SOA Deemed to be University, Bhubaneswar, INDIA. \\ ${ }^{2}$ Department of EE, IGIT Sarang, Odisha, INDIA.
}

\begin{abstract}
In the world of digital image processing, image denoising plays a vital role, where the primary objective was to distinguish between a clean and a noisy image. However, it was not a simple task. As a consequence of everyone's understanding of the practical challenge, a variety of methods have been presented during the last few years. Of those, wavelet transformer-based approaches were the most common. But wavelet-based methods have their own limitations in image processing applications like shift sensitivity, poor directionality, and lack of phase information, and they also face difficulties in defining the threshold parameters. As a result, this study provides an image de-noising approach based on Bi-dimensional Empirical Mode Decomposition (BEMD). This project's main purpose is to disintegrate noisy images based on their frequency and construct a hybrid algorithm that uses existing de-noising techniques. This approach decomposes the noisy picture into numerous IMFs with residue, which were subsequently filtered independently based on their specific properties. To quantify the success of the proposed technique, a comprehensive analysis of the experimental results of the benchmark test images was conducted using several performance measurement matrices. The reconstructed image was found to be more accurate and pleasant to the eye, outperforming state-of-the-art denoising approaches in terms of PSNR, MSE, and SSIM.
\end{abstract}

Key-Words: -image de-noising, adaptive multi-resolution, biomedical images, BEMD, PSNR, MSE, and SSIM.

Received: February 25, 2021. Revised: October 7, 2021. Accepted: November 30, 2021. Published: January 4, 2022.

\section{INTRODUCTION}

One of the fundamental challenges in the field of computer vision is image de-noising, which encourages the suppression of noise from a noise-contaminated image. In the presence of unwanted noise, image processing tasks are adversely affected [1]. Furthermore, it was observed that images are inevitably corrupted during the processes of acquisition, compression, and transmission, which leads to the loss of valuable image information. As denoising plays an important role in the application areas such as video processing, tracking, image analysis, restoration, registration, segmentation, and classification where visually pleasing images are essential, a special focus is required on it [1-2].

On account of the above factors, it may be concluded that noise removal is still a challenging task for researchers. For a better result, numerous image denoising techniques were developed afterwards, including the Spatial-domain filter, the Transformdomain filter, the Partial Differential Equation, and the Variational approach[3]. The spatial filter performs data operations directly on the pixels of the original image. Mean, median, and low pass filters were some of the most commonly used spatial-domain image denoising techniques. The spatial domain linear techniques are mathematically simple, but they have the problem of introducing blurring.

In the transform-domain image denoising approach, images are transferred from the spatial domain to the frequency domain as a preprocessing step. Then the coefficients of the image were modified using various techniques. The image was retransferred to the spatial domain using the inverse transform. After using this approach, the noises were eliminated.Some transform domain approaches include the Fourier transform, Discrete Cosine Transform, Discrete Wavelet Transform, Integer Wavelet Transform, and KL Transform. Fourier and wavelet transformations are two of the most extensively utilised image denoising methods [4-5].The Fourier transform converts an image into sine and cosine components.During the transformation process, very little information gets lost, which is the strength of the Fourier Transform. Where as the inherent tradeoff between frequency and time resolution in the Fourier transformation is a severe drawback.Later on, the wavelet transform became an alternatedue to its ability to provide better time and frequency resolution of a signal [3-7]. 
The wavelet is a time-frequency analysis method that adaptively selects the appropriate frequency band based on the signal's characteristics [37]. The frequency band is then matched to the spectrum, improving the time-frequency resolution. At this stage, many authors have applied some mathematical operation such as thresholding to suppress the noise [710]. Then denoising is accomplished by reversing the wavelet coefficients into the spatial domain. The whole process is known as the wavelet-based denoising technique [9-12]. In maximum denoising cases, wavelet thresholds are applied to remove the Gaussian noise. Soft thresholding and hard thresholding are the two most commonly used wavelet-based thresholding techniques [13]. But in the case of soft thresholding, over smoothing affects the reconstructed image. On the other hand, in hard thresholding, many coefficients are made zero. This causes blur and artifacts.Therefore, even though threshold-based image denoising methods present favourable results, the artefacts are still noticeable [14-16]. In addition, the wavelet transform has lower singularity and directional effect issues. From an operational point of view, DWT decomposes an image into a set of mutually orthogonal wavelet basis, for which a constant set of filters are used. These filters are not image-dependent. Moreover, the inverse DWT increases the computational complexity. It is quite difficult to choose a suitable mother wavelet.

When dealing with images with low noise density, the partial differential equation has a greater effect. However, when dealing with images with high noise density, the effect is poor and it takes a longer processing time. The advantages of image denoising using the total variational method are that it determines the energy function of the image. It outperforms basic approaches like linear smoothing or median filtering while smoothing out edges to a greater extent [6].

This motivated us towards an image-dependent denoising method using bi-dimensional empirical mode decomposition (BEMD) [17]. The BEMD is a timedomain approach well suited for non-linear and nonstationary signal analysis. By applying EMD, the image is decomposed adaptively into integral oscillatory components, named Intrinsic Mode Functions (IMF) and residue. In this investigation, the BEMD decomposed images are filtered using the classical filters. The key issues addressed in this study are the

$>$ Smoothing of flat regions,

$>$ Protection of edge information without blurring,
$>$ Preservation of internal texture,

$>$ Suppression of new artefacts.

To outline the paper's objective, Section 2 demonstrates the detailed methodology in algorithm form. Section 3 describes the experimental results as well as comparisons with other state-of-the-art methods with proper evidence. The conclusion and future work are given in section 4 .

\section{Methodology}

In this section, some of the fundamental issues related to image denoising with different types of noises having zero mean and finite variance are considered and their characteristics are elaborately discussed.

\subsection{Noise Model}

In digital image processing, noise is generally classified as additive or multiplicative, depending on how it is distributed. The best additive noise used in the most common type of image denoising work is Gaussian noise. The white Gaussian noise is spatially uncorrelated, which means that the noise for each pixel is independent and identically distributed. In this process, each pixel of a digital image changes by a small amount from its actual value. As a result, the image is soft and slightly blurred. Equation (1) demonstrates the additive Gaussian noise model.

$$
\mathrm{I}(\mathrm{x}, \mathrm{y})=\mathrm{M}(\mathrm{x}, \mathrm{y})+\mathrm{n}(\mathrm{x}, \mathrm{y})
$$

Where $\mathrm{I}(\mathrm{x}, \mathrm{y})$ is the noise-contaminated image function, $M(x, y)$ is the original noise-free image, and $\mathrm{n}(\mathrm{x}, \mathrm{y})$ represents the signal-independent additive Gaussian random noise with zero variance.

In some cases, noise arises due to environmental conditions such as voltage spikes in the circuits or random changes in the physical properties of materials. This kind of noise is categorised as "multiplicative noise" and is also known as "speckle" noise. The multiplicative noise model can be depicted in Equation (2).

$$
I(t)=(I-e) M(t)+n(t)
$$

Where e has a probability of $\mathrm{p}$ and lies between 0 and 1 , $I(t)$ is the noisy image at a specific time $(t), M(t)$ is the original signal, and $\mathrm{N}(\mathrm{t})$ is the speckle noise introduced during image capture, transmission, or other processing. 


\subsection{Image Decomposition}

Image decomposition is an image processing technique where the image is segregated into multiple images based on its features and frequency. In this paper, we have used frequency-based decomposition using BEMD [17]. The EMD can decompose the image into $\mathrm{n}$ levels based on the frequency of the input signal. In this model, we have used a four-level decomposition as illustrated in fig. 1 .

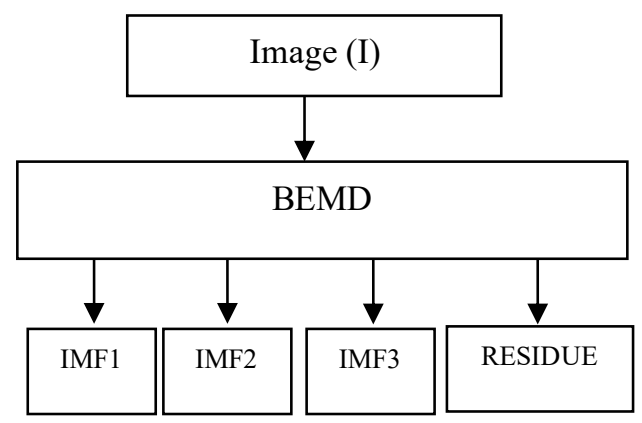

Fig. 1: Image decomposition using BEMD

\subsection{Image Denoising Model}

The proposed denoising model is presented in Figure 2.

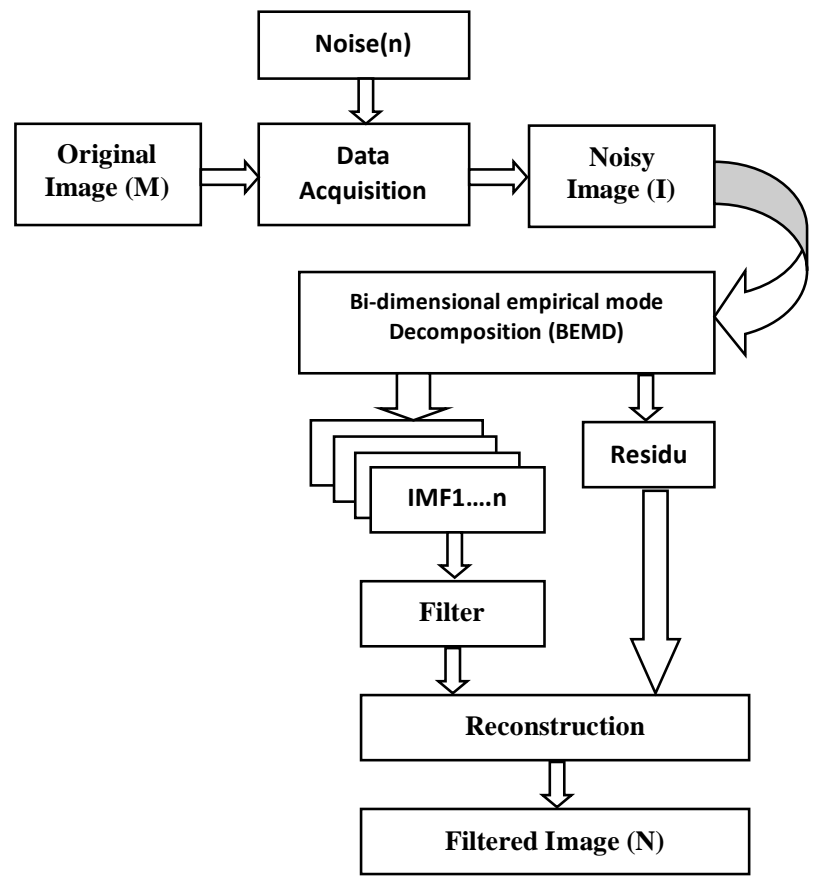

Fig.2: Image denoising using BEMD

Initially, the input image, the data acquisition system, and external sources of noise are modelled to generate a noisy image. In this experiment, Gaussian noise, salt and pepper noise, and speckle noise with zero mean and different variances are considered. The behaviour of the mentioned noises is additive or multiplicative. The noisy image is then segmented into four pieces based on their frequency using a four-level emperical mode decompsition. The breakdown images are named IMF1, IMF2, IMF3, and residue. IMF1, IMF2, IMF3 and residue maintain frequencies ranging from high to low.

The objective of this proposal is to remove noise from homogeneous areas of a noisy image while preserving structures such as edges and corners. On the other hand, considering the decomposed images separately, the valuable hidden information has to be preserved [1718]. Moreover, noise is widely known as highfrequency in nature. So the high-frequency components of digital images are filtered to eliminate the unwanted noise. Additionally, the low-frequency component contains information about hidden structures as its pixel values fluctuate slowly over time. Thus, the residual image is left unfiltered.

\subsection{Bidimensional Empirical Mode Decomposition (BEMD)}

The complicated two-dimensional model data set (Image) can be decomposed into a finite number of unique frequency components, which are known as intrinsic mode functions (IMF) [19]. These IMFs are extracted by applying a sifting process that repeats the steps until fewer than 2 maxima points occur. The uniqueness of the BEMD is similar to that of the EMD, which is used for one-dimensional signals. If I $(\mathrm{x}, \mathrm{y})$ is defined as the image which is to be decomposed into a series of BIMFs and a residue (eq.3),

$\mathrm{I}(\mathrm{x}, \mathrm{y})=\sum_{i=1}^{K} I M F_{i}(\mathrm{x}, \mathrm{y})+\operatorname{Res}(\mathrm{x}, \mathrm{y})$

Where the IMFi (x, y) is the ${ }^{\text {th }}$ IMF component. The frequency of IMF1 is higher than the other IMFs. The detailed process is demonstrated in Figure 3.

\subsection{Noise}

Noise is treated as an external energy that corrupts the signal and changes its characteristics. In digital image processing, noise may be treated as any type of random variation in brightness that changes the pixel data. This generally happens during the use of a digital camera, sensor, or scanner.

The noise generated by the electronic data acquisition systems and communication channels varies substantially, with different effects. Salt and pepper, Gaussian, and speckle noise are common sources of visual distortion. 


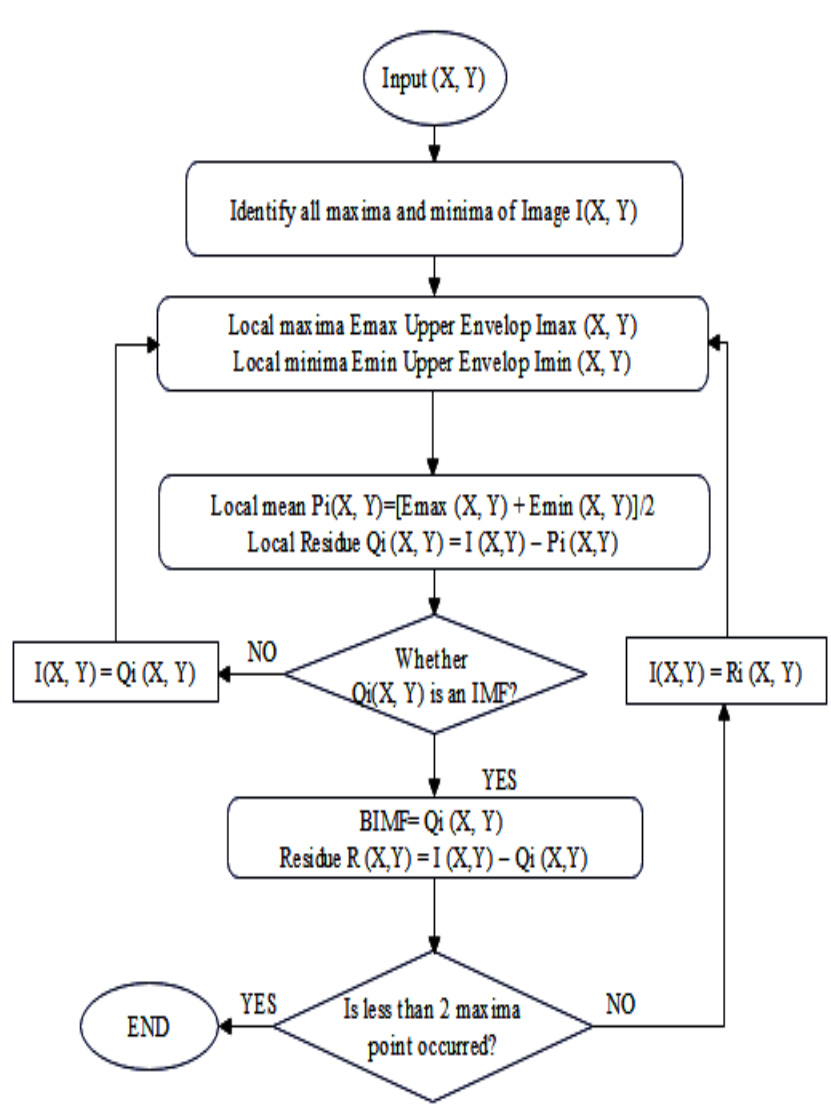

Fig. 3: Flowchart of BEMD

\subsubsection{Salt \& Pepper Noise:}

Salt-and-pepper noise is also known as impulse noise, which is a form of white and black pixel that can sometimes be seen on images. In most of the image applications for suppressing the above noise, traditional filters such as the Median filter or morphological filter are used. This type of noise is generally caused by both software and hardware (camera sensor) faults during image photographing or transmission. The probability density function "S" of a Gaussian random variable "u" is formulated as

$$
\begin{aligned}
\mathrm{S}(\mathrm{u}) & =\mathrm{S}_{\mathrm{P}} \text { for } \mathrm{u}=0 \text { (Pepper) } \\
& =\mathrm{Ssfor} \mathrm{u}=2^{\mathrm{n}}-1 \text { (Salt) } \\
& =1 \text {-(Sp-Ss) for } \mathrm{u}=\mathrm{k}\left(0<\mathrm{k}<2^{\mathrm{n}}-1\right)
\end{aligned}
$$

\subsubsection{Gaussian Noise:}

Gaussian noise is statistical noise that is identically distributed at any pair of times. Sensor noise, which is caused by temperature and poor lighting, is the primary source of Gaussian noise.

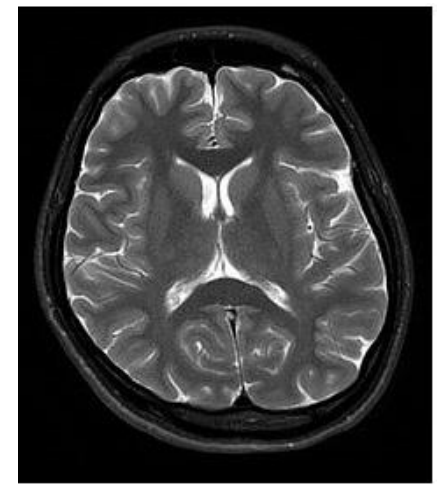

(a)

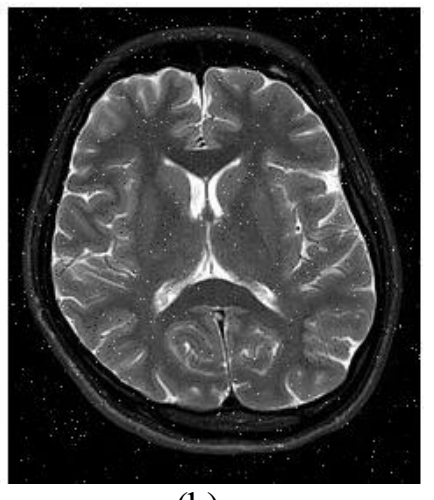

(b)
Fig. 4: (a) Input MRI brain images (b) image with Salt and Pepper noise and 0.01 variance

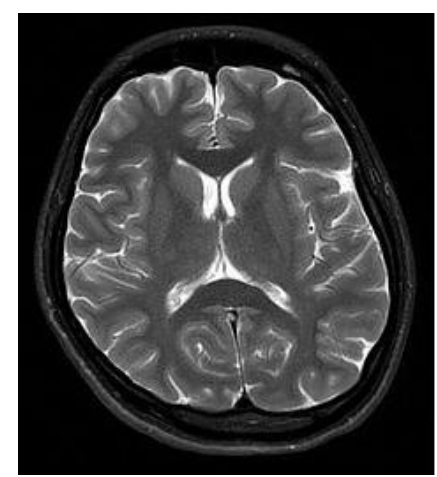

(a)

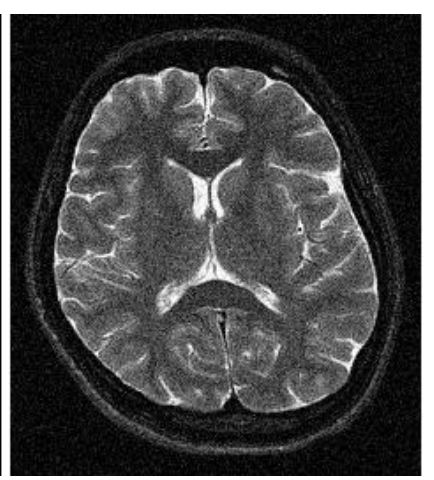

(b)
Fig. 5: (a) Input MRI brain images (b) image with Gaussian noise and 0.01 variance

Gaussian noise is reduced using spatial filters. The probability density of a Gaussian random variable is given by:

$$
h(z)=\frac{1}{\sigma \sqrt{2 \pi}} e^{-\frac{(z-\mu)^{2}}{\sigma^{2}}}
$$

Where the parameters $\mathrm{z}, \mu$ and $\sigma$ represent the graylevel, mean and standard deviation.

\subsubsection{Speckle Noise:-}

Speckle noise is modelled as a multiplicative noise that arises due to the effect of environmental conditions. This type of noise is mostly noticed in medical images and radar images. In speckle noise, the signal and the noise are statistically independent and directly proportional to the local grey level of any image area. This can be represented as follows (eq. 6)

$$
F(b)=\frac{b^{\alpha-1}}{(\alpha-1) ! a^{\alpha}} e^{\frac{-b}{a}}
$$

Where $\mathrm{b}$ is the Grey level and $a^{\alpha}$ isthe Variance. 


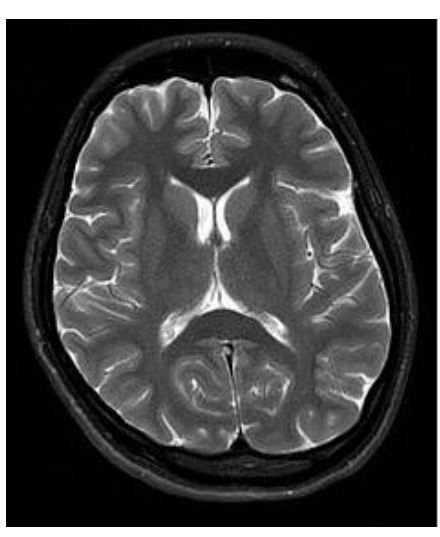

(a) (b)

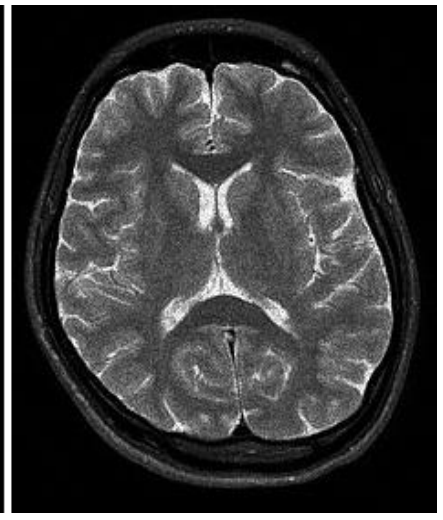

Fig. 6: (a) Input image MRI brain images (b) image with Speckle noise and 0.01 variance

\subsection{Filtering:}

Filtering is an algorithm that converts the pixel values of an image or a small part of it by applying some process. These are used for noise (unwanted artifacts) reduction, contrast enhancement, and brightness preservation purposes. Nowadays, filters are mostly used for the suppression of high-frequency components of an image. As a result, the image is smoothed and the edge is preserved.Compared with the frequencydomain, in the spatial domain, noise removal is easier because it requires much less processing time. The filters are broadly divided into two categories: (i) linear filters and (ii) non-linear. Both have some advantages as well as disadvantages. If we consider the linear filter, it has the advantage of faster processing but fails to preserve the edge. Where a nonlinear filter can preserve the edge with the compromise of processing speed.

\subsubsection{Median filter:}

The Median Filter is a non-linear filter having the ability to remove salt and pepper type noise. It uses a pre-defined window size. During the filtering process, the median filter replaces the pixel values with the median value of neighbouring pixels. Because edge information is the crucial data for an image, the median filter plays a vital role in preserving the edges during the smoothing process.

\subsubsection{Gaussian filter:}

A Gaussian filter is a linear filter whose impulse response is a Gaussian function. This filter is commonly used for smoothing, noise reduction, and computing image derivatives. Furthermore, this classic filter effectively reduces noise while significantly blurring the edges. The standard deviation used in the Gaussian function plays a vital role in its behavioural features. The two-dimensional Gaussian filter is represented as

$$
\mathrm{G}(\mathrm{p}, \mathrm{q})=\frac{1}{2 \pi \sigma^{2}} \mathrm{e}^{\frac{\mathrm{p}^{2}+\mathrm{q}^{2}}{2 \sigma^{2}}}
$$

Where $\mathrm{p}$ and $\mathrm{q}$ are the horizontal and vertical distances of the pixel from the origin. $\sigma$ is the standard deviation. A Gaussian filter reduces the contrast and preserves the brightness of the filtered image. As per its characteristics, it is designated as the ideal timedomain filter.

\subsubsection{Wiener filter:}

The Wiener filter is a stationary linear filter used for inverse filtering and noise smoothing. In inverse filtering, the filter works as a high-pass filter by using de-convolution. In compression mode, it functions as a low-pass filter to remove noise. In the process of filtering, it minimises the overall mean square error. This technique gives a better result in the case of additive white Gaussian noise (AWGN). This filter is applicable to noise having a zero mean and uses a stochastic framework to provide the linear estimation. The limitation of Wiener filtering is that it requires knowledge of the power spectra of the noise and the original image. The Wiener filter can be mathematically expressed as follows:

$$
W\left(f_{1}, f_{2}\right)=\frac{H^{*}\left(f_{1}, f_{2}\right) S_{x x}\left(f_{1}, f_{2}\right)}{\left|H\left(f_{1}, f_{2}\right)\right|^{2} S_{x x}\left(f_{1}, f_{2}\right)+S_{\tau \tau}\left(f_{1}, f_{2}\right)^{\prime}}
$$

Where, $S_{x x}\left(f_{1}, f_{2}\right)$ is the power spectral of the original image, $\mathrm{S} \tau \tau\left(f_{1}, f_{2}\right)$ is the power spectral of additive Gaussian noise, $H\left(f_{1}, f_{2}\right)$ is the blurring filter.

\section{EXPERIMENTAL RESUlTS}

The performance of the proposed method is evaluated subjectively using performance metrics such as Mean Square Error (MSE), Peak Signal to Noise Ratio (PSNR), and Structural Similarity Index Measure (SSIM). In this paper, a set of standard MRI brain images with a $256 \times 256$ size was investigated. For this study, Matlab 14a, with an Intel (R) $2.40 \mathrm{GHz}$ CPU and $4 \mathrm{~GB}$ of memory, was used. During the experiment, the original image is added with noise (salt and pepper noise, Gaussian noise, speckle noise) having different variances. The noisy images with a variance of 0.01 are shown in Figs. 4-6. The proposed denoising technique is compared with three state-of-the-art filtering methods like the Median Filter, Gaussian Filter, and Wiener filter. The comparative results are demonstrated in Fig. 7-9. 


\subsection{Metrics of Performance measures}

\subsubsection{Mean Square Error:}

The MSE represents the aggregate of the square of the error between the de-noised image and the reference image. The lower the value of MSE, the closer the two images are. The equation (9) is used for MSE calculation.

MSE $=\frac{1}{p q} \sum_{a=0}^{\mathrm{p}-1} \sum_{\mathrm{b}=0}^{\mathrm{q}-1}[\mathrm{M}(\mathrm{a}, \mathrm{b})-\mathrm{N}(\mathrm{a}, \mathrm{b})]^{2}$

Where, $p q$ : Dimension of the image.

$M(a, b)$ : Intensity of pixels $(a, b)$ of original image.

$\mathrm{N}(\mathrm{a}, \mathrm{b})$ :Intensityof pixels $(\mathrm{a}, \mathrm{b})$ after de-noising.

\subsubsection{Peak signal-to-noise ratio}

The peak signal-to-noise ratio (PSNR) is the ratio of the signal power of the processed image to the referral image. The higher value of PSNR represents a better quality of performance. PSNR is denoted as:

$$
\begin{aligned}
\operatorname{PSNR}_{\mathrm{db}} & =10 \log _{10}\left(\frac{\mathrm{MAX}^{2}}{\mathrm{MSE}}\right) \\
& =20 \log _{10}(\mathrm{MAX})-10 \log _{10}(\mathrm{MSE})
\end{aligned}
$$

MAX is the maximum possible pixel value of the image which is 255 in 8-bit image systems.

\subsubsection{Structural similarityindex}

The SSIM is a perceptual metric used for quantifying the image quality which has been degraded by the processes of data compression, data transmission, and data acquisition. It is a full-reference metric comparison method that requires a minimum of two images: the reference image and a processed image. The range of the SSIM is between -1 and 1 , to indicate the similarity. The closer the value is to one, the more similar the structure.

$\operatorname{SSIM}(M, N)=[\mathrm{q}(\mathrm{M}, \mathrm{N})]^{\alpha}[\mathrm{w}(\mathrm{M}, \mathrm{N})]^{\beta}[\mathrm{e}(\mathrm{M}, \mathrm{N})]^{\gamma}$

Where,

$$
\begin{aligned}
& q(M, N)=\frac{2 \mu_{M} \mu_{N}+C_{1}}{\mu_{M}^{2}+\mu_{N}^{2}+C_{1}} \\
& W(M, N)=\frac{2 \delta_{M} \delta_{N}+C_{2}}{\delta_{M}^{2}+\delta_{N}^{2}+C_{2}} \\
& e(M, N)=\frac{\delta_{M N}+C_{3}}{\delta_{M} \delta_{N}+C_{3}}
\end{aligned}
$$

Where,

$\mu_{M}=$ Localmeanforiamgea

$\mu_{\mathrm{N}}=$ localmeanforimageb

$\delta_{\mathrm{M}}=$ standraddeviationforimagea

$\delta_{\mathrm{MN}}=$ cross co-variance for image a,b

$\delta_{N}=$ standrad deviation for image b

If $\alpha=\beta=\gamma=1$ and $\mathrm{C}_{3}=\mathrm{C}_{2} / 2$ then the above index is simplifying to:

$\operatorname{SSIM}=\frac{\left(2 \mu_{M} \mu_{N}+C_{1}\right)\left(2 \delta_{M} \delta_{N}+C_{2}\right)}{\left(\mu_{M}{ }^{2}+\mu_{N}{ }^{2}+C_{1}\right)\left(\delta_{M}{ }^{2}+\delta_{N}{ }^{2}+C_{2}\right)}$

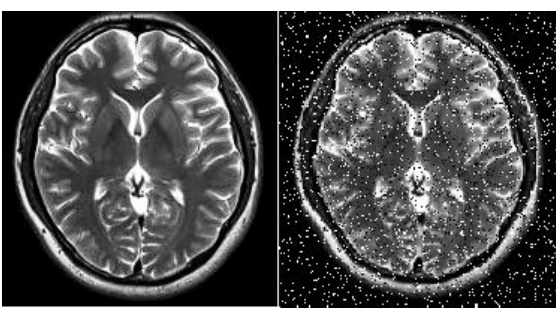

(a)

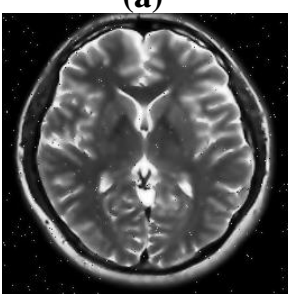

(d) (b)

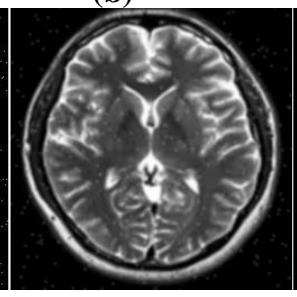

(e)

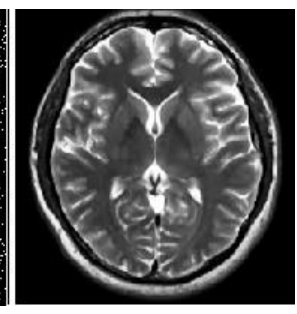

(c)

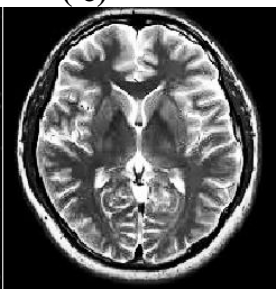

(f)
Fig.7: (a) Input MRI brain image (b) Image with Salt and pepper noise with density 0.1 (c) median filtered image (d)wiener filtered image(e) Applying Gaussianfiltered image (f) Proposed BEMD with Gaussian filter method.

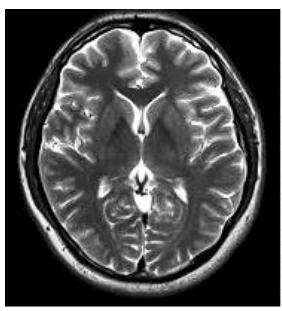

(a)

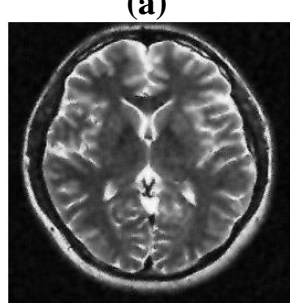

(d)

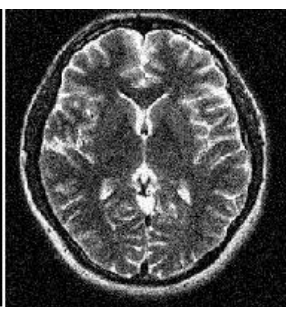

(b)

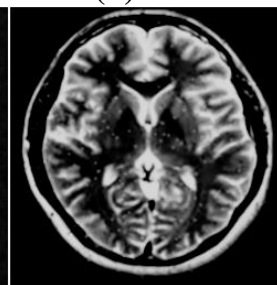

(e)

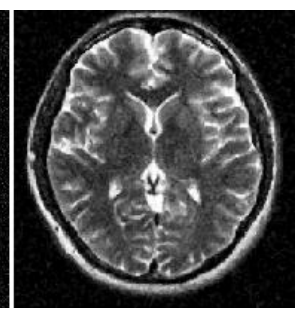

(c)

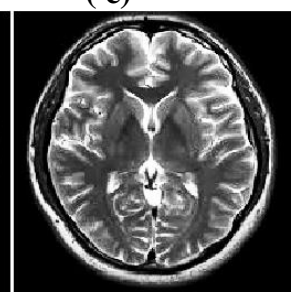

(f)
Fig. 8 (a) Input image (b) Image with Gaussian noise and variance 0.01 (c) median filtered image (d) wiener filtered image (e) Applying Gaussianfiltered image (f) Proposed BEMD with Gaussian filter method. 


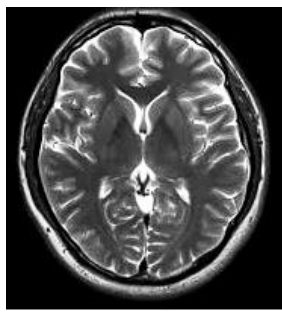

(a)

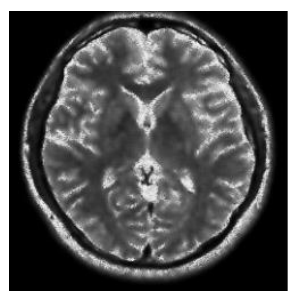

(d)

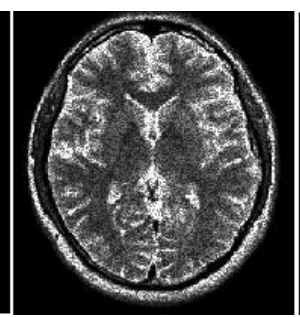

(b)

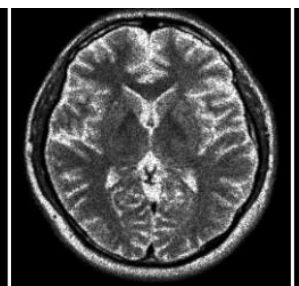

(e)

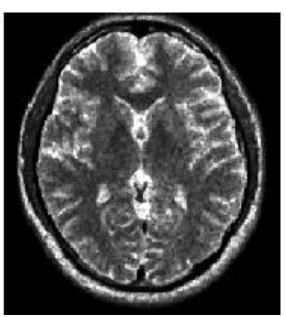

(c)

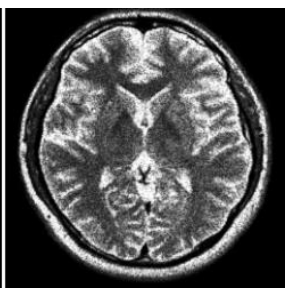

(f)
Fig. 9: (a) Input MRI brain image (b) Image with Speckle noise with variance 0.1) (c) Median filtered image (d) wiener filtered image(e) Applying Gaussianfiltered image (f) Proposed BEMD with Gaussian filter method.

\subsection{Result and Discussion}

The proposed system was verified in this section using four-level empirical mode decomposition techniques, as discussed in the previous section. MSE, PSNR, and SSIM were the three measuring parameters for each test. Table 1-3 shows the performance of each of these matrices. The range of noise variance considered in each experiment is from 0.001 to 0.1 . Figure $7-9$ depicts a few samples of MRI brain images for visual evaluation, where the images are modelled with 0.1 variance using various noises

This method incorporates the traditional filters with BEMD. Separately, the median filter with BEMD, the Gaussian filter with BEMD, and the wiener filter with BEMD are studied. In every evaluation, the hybridization outcome was found to be much better than the straight filtration procedure. In Figure 7, the brain MRI image is considered and was affected by salt and pepper noise with a density of 0.1 . The noisy image was filtered by the median filter, wiener filter, and Gaussian filter. The resulting images are presented in Figures 7 (c) to 7 (e). Figure 7 (f) illustrates the proposed BEMD with a gaussian filter. The median filter is quite good at reducing "salt and pepper" noise. After thoroughly testing the effect of salt and pepper noise, it was discovered that the proposed method outperforms the median filter (Figure 7). Experiments on Gaussian and speckle noise were also conducted, with the results displayed in Figs. 8 and 9. The comparison of PSNR values is depicted in fig. 10 as a bar graph.Separately, considering the graphs, it was found that the PSNR value of the denoised images increases when filters are applied in combination with BEMD. The graphic performance of MSE is depicted in fig. 11. The proposed method presents a lower error when a hybrid filer is applied. At present, there is no MSE value that has been fixed as a proper value. Simply put, the lower the value, the better. While the greater the MSE, the less similar they are, it will be more difficult to detect anything if the MSE between image sets differs at random. SSIM, on the other hand, uses a scale of -1 to 1 to rate everything. The structural similarity index of the proposed method as a comparison isdepicted in fig. 12.

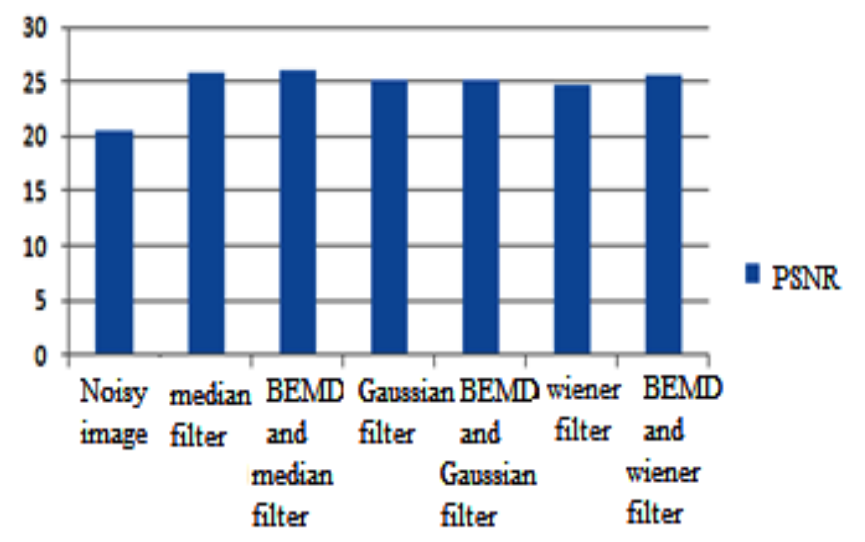

Fig. 10: Comparision of PSNR value for Gaussian noise affected image filtered by different methods and the proposed method

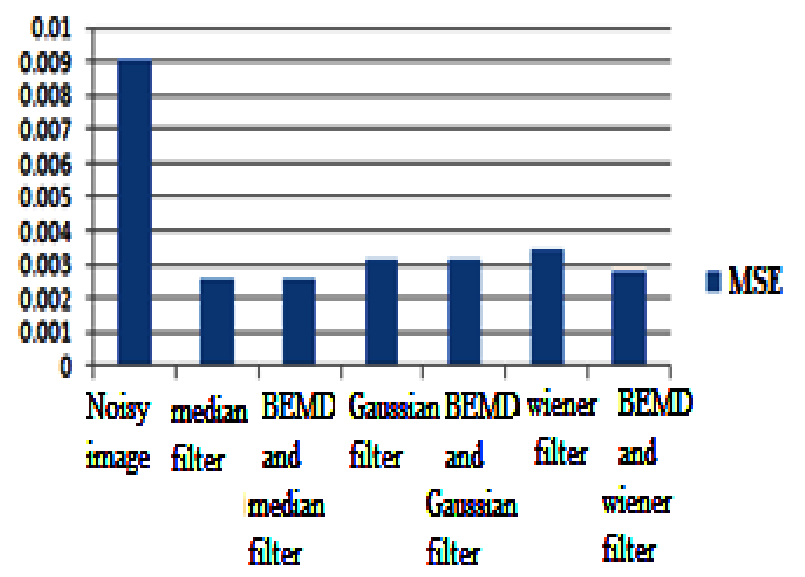

Fig. 11: Comparision of MSE value for Gaussian noise affected image filtered by different methods and the proposed method

By using equations 9,10 , and 15 , the values of MSE, PSNR, and SSIM of a set of images are calculated. The average value of each noise is recorded in Table I to Table III. Table 1 shows the Gausian noise affected image and its filtering both in the classical method and the proposed method. The result supports the claims of the proposed method numerically. 


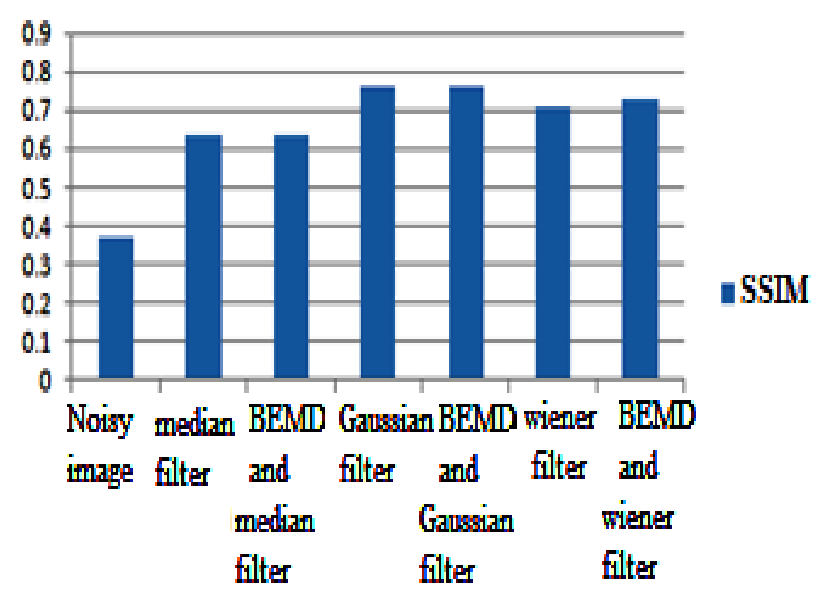

Fig.12: Comparison of SSIM value for Gaussian noise affected image filtered by different methods and the proposed method

Table 1.Comparison of PSNR, MSE, SSIM of different filters concerningthe proposed method for Gaussian noise affected image

\begin{tabular}{|l|c|c|c|}
\hline & PSNR & MSE & SSIM \\
\hline Noisy Image & 20.45507 & 0.009067 & 0.369267 \\
\hline Median Filter & 25.93197 & 0.002567 & 0.634567 \\
\hline Gaussian Filter & 25.1281 & 0.003167 & 0.763433 \\
\hline wiener filter & 24.74497 & 0.003433 & 0.708 \\
\hline $\begin{array}{l}\text { Proposed method } \\
\text { with Median } \\
\text { Filter }\end{array}$ & 25.97003 & 0.002533 & 0.636967 \\
\hline $\begin{array}{l}\text { Proposed method } \\
\text { withGaussian } \\
\text { Filter }\end{array}$ & 25.15897 & 0.003152 & 0.763467 \\
\hline $\begin{array}{l}\text { Proposed method } \\
\text { with wiener filter }\end{array}$ & 25.62447 & 0.0028 & 0.7288 \\
\hline
\end{tabular}

Table 2. Comparison of PSNR, MSE, SSIM of different filters with respect to the proposed method for Speckle noise affected image

\begin{tabular}{|l|c|c|c|}
\hline & PSNR & MSE & SSIM \\
\hline Noisy Image & 20.45507 & 0.009067 & 0.369267 \\
\hline Median Filter & 21.61433 & 0.007033 & 0.5184 \\
\hline Gaussian Filter & 23.6321 & 0.0048 & 0.742633 \\
\hline wiener filter & 23.00047 & 0.005533 & 0.6599 \\
\hline $\begin{array}{l}\text { Proposed } \\
\text { method with } \\
\text { Median Filter }\end{array}$ & 21.75607 & 0.0068 & 0.5251 \\
\hline $\begin{array}{l}\text { Proposed } \\
\text { method } \\
\text { withGaussian } \\
\text { Filter }\end{array}$ & 23.6626 & 0.004767 & 0.742367 \\
\hline $\begin{array}{l}\text { Proposed } \\
\text { method with } \\
\text { wiener filter }\end{array}$ & 23.59537 & 0.0048 & 0.675 \\
\hline
\end{tabular}

In table II, the performance of speckle noise is presented. By comparing the median filter with the bemd-median filter, it was found that the PSNR value increased by $0.65 \%$ and the MSE decreased by $3.31 \%$. In the case of the Gaussian filter and the bemd-gaussian filter, the results do not show any significant deference. Considering Table III, where salt and pepper noise are considered, the median filter gives a detrimental result. As per Z. Wang and A.C. Bovik in some cases, the MSE and PSNR give adverse results, even if the result is visually good [20].

Table 3.Comparison of PSNR, MSE, SSIM of different filters with respect to the proposed method for salt and peeper noise affected image

\begin{tabular}{|l|l|l|c|}
\hline & PSNR & MSE & SSIM \\
\hline Noisy Image & 20.45507 & 0.009067 & 0.369267 \\
\hline Median Filter & 30.27383 & 0.0019 & 0.731 \\
\hline Gaussian Filter & 23.54703 & 0.004567 & 0.681833 \\
\hline wiener filter & 22.48283 & 0.005767 & 0.610133 \\
\hline $\begin{array}{l}\text { Proposed } \\
\text { method with } \\
\text { Median Filter }\end{array}$ & 27.53493 & 0.001833 & 0.740333 \\
\hline $\begin{array}{l}\text { Proposed } \\
\text { method } \\
\text { withGaussian } \\
\text { Filter }\end{array}$ & 23.56583 & 0.0045 & 0.682 \\
\hline $\begin{array}{l}\text { Proposed } \\
\text { method with } \\
\text { wiener filter }\end{array}$ & 22.82073 & 0.0053 & 0.614733 \\
\hline
\end{tabular}

\section{Conclusion}

This paper has analysed the process of noise generation and the characteristics of common image noises, studied the different filtering techniques used in the spatial and transfer domains, discussed the methods to choose the structural decomposition technique, studied the fundamental theories of empirical mode decomposition (EMD) and its mathematical morphology, exploited the advantages of empirical mode decomposition with a multi-resolution structure, investigated the selection criteria for filters, and proposed a filtering algorithm with structural decomposition combined with image denoising. Experiments on a set of benchmark images demonstrate that the proposed technique outperforms similar types of denoising algorithms, particularly in terms of PSNR, MSE, SSIM index, and visual effect.

The future work of this paper is as follows:

(1) Before filtration, a threshold factor is applied to the coefficients of each decomposed image.

(2) The threshold factors must be noise-dependent. 
(3) A new algorithm will be proposed, combining BEMD with soft computing techniques (deep learning, fuzzy logic, artificial neural networks, genetic algorithms, particle swarm optimization algorithms) to improve the denoising performance.

\section{References:}

[1] GonzalezR.C, WoodsR.E. Digital Image Processing. Englewood Cliffs, NJ:Prentice-Hall, 2007.

[2] Mallat S. A. wavelet tour of signal processing: the sparse way 3rd edition. Academic Press, Elsevier, Burlington, 2008.

[3] Jinjuan Wang, Shan Duan, Qun Zhou, An Adaptive Weighted Image Denoising Method based on Morphology, International Journal of Circuits, Systems and Signal processing, 2021; 15: 271-279.

[4] Gupta D., Ahmad M. Brain MR image denoising based on wavelet transform. Int. J. Appl. Technol. Eng. Explor, 2018, 5(38): 11-16.

[5] Shukla U. P., Nanda S. J. Denoising Hyperspectral Images Using Hilbert Vibration Decomposition With Cluster Validation, IET Image Processing, 2018; 12 ( 10):1736-1745.

[6] Green M., Marom E.M. , Konen E., et al. Patientspecific image denoising for ultra-low-dose CTguided lung biopsies. Int J Comput Assist Radiol Surg. 2017;12(12):2145-2155.

[7] Ellinas J. N., Mandadelis T., Tzortzis A., et al. Image de-noising using wavelets. T.E.I Piraeus Appl. Res. Rev.,2004,IX(1): 97-109

[8] Zhang X. Image denoising using dual-tree complex wavelet transform and wiener filter with modified thresholding. J Sci Ind Res India, 2016 ;75(11):687690.

[9] Fedak V, Nakonechnyy A. Adaptive wavelet thresholding for image denoising using SURE Minimization and Clustering of Wavelet Coefficients. Technical Transaction on Electrical Engineering, 2015: 197-210.

[10] Kimlyk M and Umnyashkin S. Image denoising using discrete wavelet transform and edge information. IEEE Conference of Russian Young Researchers in Electrical and Electronic Engineering (EIConRus), 2018: 1823-1825,

[11] Bnou K, Raghay S and Hakim A. A wavelet denoising approach based on unsupervised learning model. EURASIP J. Adv. Signal Process. 2020, 36.

[12] Sameera V. Mohd Sagheer., Sudhish N. George, A review on medical image denoising
algorithms,Biomedical Signal Processing and Control. 2020(61).

[13] Donoho D. L. De-noising by soft-thresholding, in IEEE Transactions on Information Theory. 1995, 41(3) : 613-627.

[14] QiC., Li Q. (2016). The improved method of wavelet denoising for nonlinear signal, Manufacturing Automation. 2016(38):14-17.

[15] Srivastava M., Anderson C. L, Freed J. H.A new wavelet denoising method for selecting decomposition levels and noise thresholds, IEEEAccess Practical Innovations Open Solutions,2016( 4): 3862-3877.

[16]Chang S. G, Yu Bin and Vetterli,M.Adaptive wavelet thresholding for image denoising and compression, in IEEE Transactions on Image Processing,2000, 9(9):1532-1546.

[17] HuangN. E, Shen Z. and LongS. et al..The empirical mode decomposition and the Hilbert spectrum for nonlinear and nonstationary time series analysis. Proc. R. Soc. London A,1998(454):903-995.

[18] Satapathy L. M, Dalai A, SatapathyS. and JenaA. Satellite image enhancement based on multitechnology fusion, 2018 Second International Conference on Inventive Communication and Computational Technologies (ICICCT), Coimbatore, 2018: 1677-1680.

[19] Dong W, Li X, Lin X and Li Z. A Bidimensional Empirical Mode Decomposition Method for Fusion of Multispectral and Panchromatic Remote Sensing Images. Remote Sensing.2014,(6):8446-8467.

[20]Wang $Z$ and Bovik A. C. Mean squared error: Love it or leave it? A new look at Signal Fidelity Measures, in IEEE Signal Processing Magazine, 2009,26(1): 98117.

\section{Contribution of individual authors to the creation of a scientific article (Ghostwriting Policy)}

L.M. Satapathy carried out conceptual framework; system design, analysis, simulations, discussion, writing, proofreading and editing.

Prof. P. Das Supervised the project.

Sources of funding for research presented in a scientific article or scientific article itself

There is no funding source for this project.

\section{Creative Commons Attribution License 4.0 (Attribution 4.0 International , CC BY4.0)}

This article is published under the terms of the Creative Commons Attribution License 4.0

https://creativecommons.org/licenses/by/4.0/deed.en_US 